\title{
El papel de las tecnologías cívicas en la redefinición de la esfera pública
}

\author{
José Manuel SÁNCHEz DuARTE \\ Universidad Rey Juan Carlos \\ josemanuel.sanchez@urjc.es \\ María Victoria Bolaños HuerTas \\ Universidad Rey Juan Carlos \\ v.bolanos@alumnos.urjc.es \\ Raúl Magallón Rosa \\ Universidad Carlos III de Madrid \\ raul.magallon@uc3m.es \\ Victoria ANDERICA CAFFARENA \\ Ayuntamiento de Madrid \\ victoriaanderica@gmail.com
}

Recibido: 30 de septiembre de 2015

Aceptado: 16 de noviembre de 2015

\begin{abstract}
Resumen
Las deficiencias de la democracia representativa, expresadas en términos de desafección y distancia con los actores políticos tradicionales, repercuten en la demanda de estructuras más accesibles a la ciudadanía que promuevan iniciativas de transparencia y favorezcan la rendición de cuentas en el ejercicio del poder. Las tecnologías cívicas, herramientas impulsadas principalmente por organizaciones de la sociedad civil, pretenden implicar a la ciudadanía en la toma de decisiones públicas. En el siguiente artículo reflexionamos sobre las potencialidades y límites de estas tecnologías en el contexto de una esfera pública dinámica profundizando en los nuevos patrones de relación entre los medios de comunicación, las redes sociales y este tipo de iniciativas.
\end{abstract}

Palabras clave: tecnologías cívicas; activismo de datos; ciberactivismo; esfera pública; redes sociales.

\section{The role of civic technologies in the redefinition of the public sphere}

\begin{abstract}
The shortcomings of representative democracy, expressed in terms of distance and disaffection with traditional political actors, have triggered the demand for new structures that are accessible to citizens and promote transparency and accountability in the exercise of power. Civic technologies, tools that are mainly driven by civil society organizations, aim to involve citizens in public decision-making processes. In the following article we reflect on the potential and limits of these technologies in a context of a dynamic public sphere with the aim of deepening the relationship between new media, social networks and civic technologies.
\end{abstract}

Keywords: civic technologies; data activism; cyberactivism; public sphere; social networks. 


\section{Referencia normalizada}

Sánchez Duarte, J.M.; Bolaños Huertas, M.V.; Magallón Rosa, R. y Anderica Caffarena, V. (2015). El papel de las tecnologías cívicas en la redefinición de la esfera pública. Historia y Comunicación Social. Vol 20, número 2, páginas 483-498.

Sumario: 1. Introducción. 2. Redefinición de la esfera pública: nuevos medios, redes sociales y sociedad civil. 2.1. Deficiencias y alternativas a la esfera pública. 2.2. Nuevos medios. De la audiencia pasiva a la capacidad generativa de los públicos. 2.3. Redes sociales como herramientas para la participación de la ciudadanía. 2.4. Patrones de relación entre los nuevos medios y la sociedad civil. 3. Tecnologías cívicas. 3.1. Definición, objetivos y categorías en las tecnologías cívicas. 3.2. Surgimiento y evolución de las tecnologías cívicas en España. 4. Periodismo y tecnologías cívicas. 5. Conclusiones. 6. Referencias bibliográficas. 7. Notas.

\section{Introducción}

En los últimos años han proliferado organizaciones de la sociedad civil centradas en la apertura de datos y la transparencia. De forma autónoma y coordinada han demandado la aprobación de una ley de transparencia en línea con los estándares internacionales. La exigencia de una apertura de datos públicos se vincula con la necesidad de participar en la definición de políticas públicas así como con la creación de herramientas (en especial digitales) que conecten a la ciudadanía con las instituciones y fomenten la participación.

En este contexto, las tecnologías cívicas se presentan como el conjunto de herramientas para la resolución de problemas pero también como plataformas y dispositivos para la acción de la ciudadanía. El objetivo de este artículo se centra en analizar la evolución de este tipo de tecnologías en España, su función dinamizadora en la esfera pública y su relación con el periodismo y las reivindicaciones de la ciudadanía.

La tradicional dependencia de los movimientos sociales de los medios de comunicación han marcado los espacios y tiempos de acción de estas organizaciones. La construcción de la agenda mediática supone un escenario de poder y disputa de los grupos sociales y los intereses económicos y políticos por incidir en las decisiones y en los marcos de interpretación de la ciudadanía (Macassi, 2009). Los gobernantes querrán atender a los problemas visibilizados por los medios a la vez que establecen relaciones (a veces simbióticas) con aquellos que ejecutan el poder.

Como contraposición, la agenda de la ciudadanía está elaborando nuevos canales de influencia en el ámbito digital. El desarrollo de la tecnología, la fuerte implantación de las redes sociales, la creación de nuevos espacios de comunicación (blogs, webs) el desarrollo de plataformas destinadas a potenciar las campañas activistas y la evolución de los medios digitales hacia plataformas más participativas permiten un mejor acceso a la esfera pública.

Si bien estos espacios y herramientas no han desplazado el papel predominante de los medios para posicionar temas en el debate público, su proliferación condiciona cada vez más las lógicas informativas. La interactividad con la audiencia está pasando 
de una posición puramente participativa (valorando noticias, insertando comentarios, notificando errores compartiendo noticias) a una interactividad productiva (Masip y Suan, 2014).

En este escenario, y de manera especial en los medios digitales, han aparecido espacios destinados a campañas y demandas ciudadanas que sustituyen en parte la intermediación de los medios e innovan en los temas sobre los que reparar. La ciudadanía desarrolla procesos cooperativos de información, impulsando campañas que más tarde se convertirán en noticias, proporcionando datos como materia prima a las informaciones, enviando fotos y audios o fabricando mapas colaborativos de denuncia.

En este proceso destaca el nuevo perfil de la ciudadanía organizada, una sociedad civil convertida en agente de producción informativa (ampliando enfoques, atribuyendo responsabilidades, etc.) que por medio de la tecnología complementa la acción y la reflexión con la producción de datos. A continuación exponemos los cambios apreciados en el ámbito de actuación de estos actores y sus patrones de relación.

\section{Redefinición de la esfera pública: nuevos medios, redes sociales y sociedad civil}

La esfera pública, concebida como un espacio de relación y mediación entre la ciudadanía y el estado, muta su naturaleza a través de los procesos globales y del desarrollo tecnológico. Este nuevo ámbito de actuación combina la acción colectiva clásica de la sociedad civil con patrones distintos de comunicación entre gobernantes y medios (Flanagin, Stohl y Bimber, 2006: 30), en los que la noción de política es cada vez mayor y más amplia (Sousa, Pinto y Costa e Silva, 2013: 10).

Se pueden distinguir tres escenarios posibles a partir de estas transformaciones: un nuevo marco de actuación entre medios, ciudadanía y representantes, un contexto similar replicando en la actualidad las deficiencias anteriores y la incertidumbre asociada al marco digital.

\subsection{Deficiencias y alternativas a la esfera pública}

La crisis democrática en términos de falta de respuesta de los gobernantes a las demandas de los ciudadanos radica en los límites y contradicciones del espacio en el que ambos actores (instituciones y ciudadanía) se relacionan e intercambian puntos de vista. Un espacio democrático en el que los intereses públicos, las opiniones, las agendas y los problemas deberían formarse, transformarse e intercambiarse por una participación ciudadana proactiva (Robertson, Vatrapu y Medina: 2010, 13). El concepto tradicional de esfera pública se cuestiona principalmente en términos de legitimidad y eficacia. En primer lugar la erosión de la legitimidad se deriva de la 
exclusión histórica de determinados grupos de la gestión del poder. Por su parte, la crítica a la eficacia radica en la imposibilidad del público para comunicar su voluntad a las instituciones y en la incapacidad de estas para realizar la voluntad del público (Fraser cit. Goldberg, 2010).

La ausencia de comunicación reside en el contacto deficiente entre representantes y representados. Los grandes medios y los estados condicionan el "mercado de las ideas" inclinando la balanza a favor de grandes corporaciones. El depósito de intercambio de información que debería ser la esfera pública se ha convertido en algo limitado (Iosifidis, 2011: 627) y sesgado a los intereses de gestores y periodistas. Esta noción ratifica la idea del declive de la esfera pública expuesta por Habermas por la cual los medios de comunicación han comercializado la esfera pública convirtiéndola en un espacio donde se da prioridad a los objetivos de la retórica y las relaciones públicas y la publicidad (Habermas, 2004).

Se considera fundamental para una democracia fuerte una esfera pública que permita la expresión de las diversas opiniones sobre cualquier tema, la constitución de servicios de comunicaciones orientados a los ciudadanos, el escrutinio del poder y, en última instancia, la soberanía pública (Dahlberg, 2007: 826). Ante la incapacidad de desarrollar estas funciones la ciudadanía dirige su atención e intención política al margen del sistema parlamentario redefiniendo la vida política y a menudo en el contexto de movimientos sociales (Dalhgren, 2005a: 155) lejos de la crítica generalizada a la apatía y pasividad de los ciudadanos.

Esta visión de la política contradice la existencia de una sola esfera pública resaltada por Habermas. Las nuevas formas de participar, las innovaciones sociales de la ciudadanía, sus espacios, lenguajes y protocolos describen la esfera pública como una constelación de espacios comunicativos que permiten la circulación de otras ideas (Dalhgren, 2005b: 148). Unos espacios periféricos (con lógicas horizontales, críticas y con incentivos a la participación) que intenta influir de manera intermitente en una esfera pública central dominada por las instituciones más asentadas de la sociedad (Sampedro, 2000).

Los ritmos y los modos de esa constelación de espacios rompen con el ideal habermasiano del discurso público racional y el consenso obligatorio. De acuerdo con una visión más pragmática, el consenso debe ser entendido como un ideal regulativo más que como una meta realista de deliberación (Grounlund, Strandberg y Himmelroos, 2009: 189). Así las organizaciones de la sociedad civil, los movimientos sociales y colectivos activistas permitirían cierta anarquía y desacuerdo reconociendo el pluralismo y la diferencia en unas sociedades en las que el estilo "racional" (y los espacios en los que se practica) suelen pertenecer a las identidades más hegemónicas (Loader y Mercea, 2011: 758).

Todos estos cambios a nivel estructural en la esfera pública se han amplificado con el desarrollo tecnológico. Internet posibilita el desarrollo de herramientas y soportes que pueden suplir deficiencias de la actividad política tradicional (Sampedro, Sánchez-Duarte y Campos, 2014: 78). La distancia con el sistema expresada en 
términos de abstención o cinismo político encontraría un campo de acción en el que experimentar nuevas prácticas destinadas a mejorar el proceso democrático. Aquellos ciudadanos más activos verían reforzado su compromiso cívico al encontrar formas más amplias y plenas de participación, así como ampliado su conocimiento e información con el surgimiento de nuevas plataformas mediáticas.

2.2. Nuevos medios. De la audiencia pasiva a la capacidad generativa de los públicos

El nuevo ecosistema mediático ha modificado los modelos tradicionales de emisión, edición y reproducción de la información. La llegada de nuevas herramientas, técnicas y formatos, así como el aumento de competencias comunicativas por parte del público han redefinido el sistema tradicional que distinguía entre productores de noticias, anunciantes y 'audiencias'. La lista de tareas que un periodista puede realizar aumenta por la plasticidad de la tecnología modificando tanto las capacidades para presentar la información como el comportamiento de la audiencia (Anderson, Bell y Shirky, 2012).

En paralelo al cambio estructural de las organizaciones periodísticas, los públicos activos asumen parte del proceso de creación, distribución y reinterpretación de la información. El periodista no es sustituido pero se sitúa en otra posición de la cadena informativa. Su papel de verificador de la información se combina con el de organizador y gestor de los flujos comunicativos producidos por los públicos (textos, enlaces, audios, fotos, vídeos...).

De igual modo y junto a la capacidad que tienen los nuevos medios de crear productos especializados para una audiencia cada vez más segmentada, los profesionales del periodismo deberían desarrollar estrategias para involucrar a nuevos públicos en las conversaciones en torno a los contenidos y generar un interés en audiencias que a priori no forman parte del destinatario inicial del medio.

Sin embargo, la interactividad productiva implica algo más que la participación de la audiencia en el proceso comunicativo y en los intentos de crear una agenda mediática particular al margen de la agenda política. El reto de la interactividad reside en integrar a ese público en el mantenimiento económico del medio. La dependencia tradicional entre medios de comunicación y anunciantes ha propiciado en ocasiones (a través de los llamados factores de preagenda) una falta de independencia de los periodistas a la hora de publicar informaciones relacionadas con los propios anunciantes (Díaz Nosty, 1995). Lo mismo ha ocurrido con la publicidad institucional a través de los actores políticos y la viabilidad de los medios (locales y regionales principalmente).

El modelo de sostenibilidad de los nuevos medios tiene clara la necesidad de diversificación como una forma de proteger su independencia. A la financiación clásica pueden unirse otras fuentes de ingresos, tanto provenientes de patrocinios o fundaciones como de la propia audiencia (por ejemplo, a través de campañas de crowdfunding). El objetivo último reside en que frente a la opacidad en la gestión y administración de los grandes medios de comunicación, las nuevas plataformas busquen otro 
tipo de modelo más abierto. Este ejercicio de transparencia no solo es un elemento fundamental en todo sistema de confianza, si no que permite reconocer otros valores ligados a la independencia y a las agendas ciudadanas.

De este modo, se crean estructuras descentralizadas que colaboran, pero también se oponen a las formas de jerarquía tradicional. Aparecen así distintas posibilidades de participación por parte de la audiencia, nuevas narrativas que redefinen al intermediario y transforman el sistema de confianza. La capacidad reactiva de la esfera pública puede convertirse en una capacidad generativa (Benkler, 2015: 266) desplazando los papeles limitados de la audiencia hacia otros perfiles más flexibles, autónomos y cooperativos y a partir de nuevos canales de acceso como las redes sociales.

\subsection{Redes sociales como herramientas para la participación de la ciudadanía}

Las redes sociales son actualmente los entornos socio-técnicos que permiten de una manera más efectiva el discurso en la esfera pública (Robertson, Vatrapu y Medina, 2010: 29). Su uso masivo e intensivo las sitúa en un lugar preeminente en la configuración y generación del debate público desde dos vías: a partir de su posición como complemento y extensión de los nuevos medios y como canal de desarrollo, comunicación y difusión de la ciudadanía.

En relación a los medios de comunicación, los públicos adquieren una mayor capacidad de participación en el proceso informativo conectando directamente con los promotores informativos a partir de espacios desintermediados en los que audiencias y fuentes se encuentran. A partir de las redes el proceso de feedback se materializa al tiempo que desarrollan el papel distribuidor de los públicos convertidos en gatewatchers. Si los medios transmiten sus noticias de forma unidireccional a sus audiencias, estos nuevos públicos redistribuyen la información a una comunidad de seguidores (Bruns, 2003). Las posibilidades de acceso a la esfera pública (al menos de recepción de información e intercambio de opiniones) se amplían y personalizan variando el consumo desde la proactividad a la recomendación. Por otra parte los movimientos sociales y la ciudadanía organizada emplean las redes sociales como una nueva plaza pública en la que comunicar acciones, difundir convocatorias o incluso crear comunidades.

Estos procesos tienen importantes influencias. Quizá efectos de las redes sociales no sean tan tangibles como el activismo práctico y convencional. Sin embargo, pueden facilitar el conocimiento de los problemas a una escala mucho más elevada (Rotman, Preece, Vieweg et. Al. 2011: 821) e incluso aumentar el compromiso cívico y la participación en la resolución de los problemas de las comunidades al incrementar los mecanismos de elaboración y reflexión de la información (Zúñiga, 2011).

Este enfoque se rebate desde una visión crítica de la relación entre redes sociales y esfera pública disminuyendo los efectos positivos derivados de la conversación y acción social en estas plataformas. Las redes sociales tienden a fomentar dinámicas de exhibición y refuerzo de la identidad de grupo. Como indica Evgeny Mozorov es poco probable que estas campañas de autopromoción sean capaces de desarrollar 
empatía o impulsar los sacrificios que la vida política requiere (2011: 187). Si bien internet no ha hecho más que digitalizar procesos que ya existían previamente, las redes sociales han amplificado este tipo de comportamientos de una manera inflexible y cínica (Lanier, 2011: 98). Como consecuencia se estaría generando un activismo de segunda categoría, incapaz de avanzar sus demandas en los espacios y procesos políticos tradicionales (Shulman cit. Christensen \& Bengtsson: 2011: 900). Las redes sociales sin la articulación de la sociedad civil y la difusión de los nuevos medios perderían parte de su potencial como dinamizadoras de la esfera pública.

\subsection{Patrones de relación entre los nuevos medios y la sociedad civil}

Una de las líneas comunes de muchos de estos nuevos medios consiste en integrar el discurso de la sociedad civil en la cadena informativa. Frente al monopolio de los temas provenientes de la política más institucional, las nuevas plataformas mediáticas ceden espacios para una construcción de los temas desde los marcos de interpretación de la ciudadanía. Así surge un modelo híbrido en el que los medios adquieren una posición militante en relación a determinados temas y los activistas encuentran espacios mediáticos para desarrollarlos; espacios de los que antes no disponían.

En el caso español, y siguiendo esta tendencia, destacan campañas como la impulsada por Infolibre en la plataforma Change con el objetivo de que las compañías eléctricas no fichen a exresponsables políticos, el blog Desalambre de eldiario.es sobre derechos humanos o el seguimiento en streaming de manifestaciones, desahucios, etc. Desde la óptica de los medios convencionales es reseñable el proyecto Planeta Futuro, un portal sobre desarrollo sostenible lanzado por El Pais, en colaboración con la Fundación Bill y Melinda Gates.

Desde una posición crítica, la proximidad de la sociedad civil con estos nuevos medios implica cierto riesgo de polarización política (en especial en países con democracias menos consolidadas). Dicha polarización tiene su origen en la fuerte fragmentación de una audiencia que se comporta como "caja de resonancia" favoreciendo la auto-referencialidad y pudiendo estar menos disponible para establecer colaboraciones y coaliciones con otros grupos (Mancini, 2013: 56). Este hecho se acrecentaría no solo con la segmentación comercial del sistema de medios de comunicación, sino también con la lógica relacional de la propia red (Sunstein, 2007).

Como contraposición a este punto de vista, esta relación apuesta por convertir a los medios en espacios de redefinición colectiva de la opinión pública, más como dinamizadores que como líderes de opinión tradicionales. Este hecho, unido a la aparición de nuevos medios puede interpretarse como un aumento de la pluralidad informativa en la medida en que permite crear una 'nueva cultura' en torno a la audiencia que se aproxima a estos medios.

En definitiva, estaríamos hablando de patrones de relación entre medios y sociedad civil con rasgos de lobby ciudadano (Anderica, 2014: 75) a través de una defensa organizada de intereses y agendas particulares (Politikon, 2014: 212) Las relaciones estables y permanentes con estos nuevos medios implica la visibilidad tanto de temas 
ignorados, como de las nuevas herramientas para la fabricación, visualización y construcción de datos y recursos empleadas desde la sociedad civil.

\section{Tecnologías cívicas}

La acción política ha alterado sus rasgos habituales con el desarrollo tecnológico. No solo a nivel de las formas más institucionalizadas de participación, sino también en el ámbito de la ciudadanía organizada. El uso de herramientas digitales puede aumentar la capacidad de las instituciones para establecer un mayor contacto y cercanía con los ciudadanos ampliando y renovando el compromiso democrático. Sin embargo, en muchos casos las innovaciones en el empleo de la tecnología aplicadas a la participación política provienen en exclusiva de las organizaciones de la sociedad civil. Su acción pasa por concebir estas herramientas como la base y el soporte de su estrategia (Van Laer y Van Aelst, 2010) así como método para disminuir las carencias y exclusiones de la esfera pública central, acceder a ella y obtener visibilidad de sus causas y reivindicaciones.

\subsection{Definición, objetivos y categorías en las tecnologías cívicas}

Por tecnologías cívicas se pueden definir aquellas herramientas, plataformas, aplicaciones e infraestructuras digitales impulsadas por organizaciones de la sociedad civil, instituciones y empresas con el objetivo de implicar a la ciudadanía en la toma de decisiones públicas (Bolaños et alt, 2015). Entre sus objetivos destacan la voluntad de aumentar la información facilitando herramientas de participación y empoderamiento de la ciudadanía, fortalecer la rendición de cuentas de los gobernantes y la transparencia en su gestión, ofrecer y mejorar los servicios públicos y, por tanto, sanear el funcionamiento democrático de las instituciones. En definitiva, este tipo de herramientas pueden mejorar las estructuras políticas y ofrecer nuevas oportunidades de participación con el objetivo de integrar a las instituciones públicas en un nuevo proceso deliberativo (Magallón, 2014).

Además del intento por restablecer de una manera eficiente el diálogo entre ciudadanía y estado las tecnologías cívicas permiten una interacción más eficiente entre ciudadanos. En un proceso de inteligencia colectiva (tangible y replicable) definen nuevos caminos de organización social más colectivos a partir de un desarrollo tecnológico de código abierto, licencias libres y resultados cívicos, muchas veces financiados desde plataformas de crowdfunding.

Según Ana Trejo (2013) este tipo de tecnologías, integradas en el denominado software cívico (o hacking cívico) tiene diversos impactos no solo en el ámbito de la participación, la transparencia y el buen gobierno. Su potencialidad reside en la posibilidad de generar espacios urbanos más sostenibles, mejorar la calidad de vida y la salud de sus habitantes, solucionar problemas de exclusión social o definir otras formas de generación de riqueza y empleo. 
Por tanto, un acercamiento a las tecnologías cívicas implica reparar no solo en la acción política sino también en la cultural y social, así como en las las relaciones establecidas dentro de cada comunidad. En este proceso, Patel, Sotsky, Gourley y Houghton (2013) categorizan las herramientas cívicas como aquellas que se ocupan de (1) datos gubernamentales (acceso a datos públicos y transparencia), las que se centran en el (2) consumo colaborativo (intercambio peer-to-peer de bienes y servicios), las plataformas y prácticas de (3) crowdfunding (proyectos que mejoren servicios públicos y espacios), las (4) redes sociales (espacios basados en un lugar y en los foros de una comunidad) y, por último (5) la organización de comunidades en las que las tecnologías cívicas reparan en causas sociales e intentan fortalecer el compromiso cívico.

\subsection{Surgimiento y evolución de las tecnologías cívicas en España}

El surgimiento de las tecnologías cívicas en España ha crecido de manera exponencial en los últimos cuatro años. Se identifican diversas causas relacionadas con el contexto político y las nuevas prácticas de acción política. La primera de ellas indica las manifestaciones, concentraciones y acampadas en torno al movimiento 15-M como un punto de inflexión en el desarrollo de estas herramientas. Muchas de las iniciativas próximas al software cívico surgieron a partir de estos acontecimientos o experimentaron un crecimiento y una relevancia significativa. El 15 de mayo de 2011 supuso un semillero de iniciativas tecnopolíticas que recogían las lógicas de prácticas previas y las adaptaban a la situación ampliando los repertorios de participación política y repolitizando segmentos sociales insatisfechos con los réditos políticos del sistema (Politikon, 2014: 205).

De igual modo, y relacionado con ese proceso de adaptación se enclava otra de las causas. Desde 1998 y con el desarrollo de los movimientos antiglobalización los movimientos sociales experimentaron un proceso continuo de internacionalización. El surgimiento de una sociedad civil transnacional (Tarrow, 2011) implicó un continuo intercambio de experiencias de participación que con el desarrollo tecnológico avanzó a un ritmo más elevado. Los repertorios de acción colectiva adquirieron una mayor modularidad replicándose en cualquier parte del mundo. En el caso de las tecnologías cívicas este tipo de alianzas (más comunicativas y de difusión que estratégicas) supusieron replicar un campo y protocolos de acción que en España estaban sin explotar.

Pese a la existencia de experiencias previas al 2011 como Discursia ${ }^{1}$ la eclosión de las tecnologías cívicas en España se ha producido con especial intensidad entre 2012 y 2014 (gráfico 1). A día de hoy existen más de 100 herramientas operativas en red que por lo general buscan proporcionar información pública al servicio de la ciudadanía y abrir nuevos canales de participación. 
Gráfico 1: Evolución de las tecnologías cívicas en España (2011-2015)

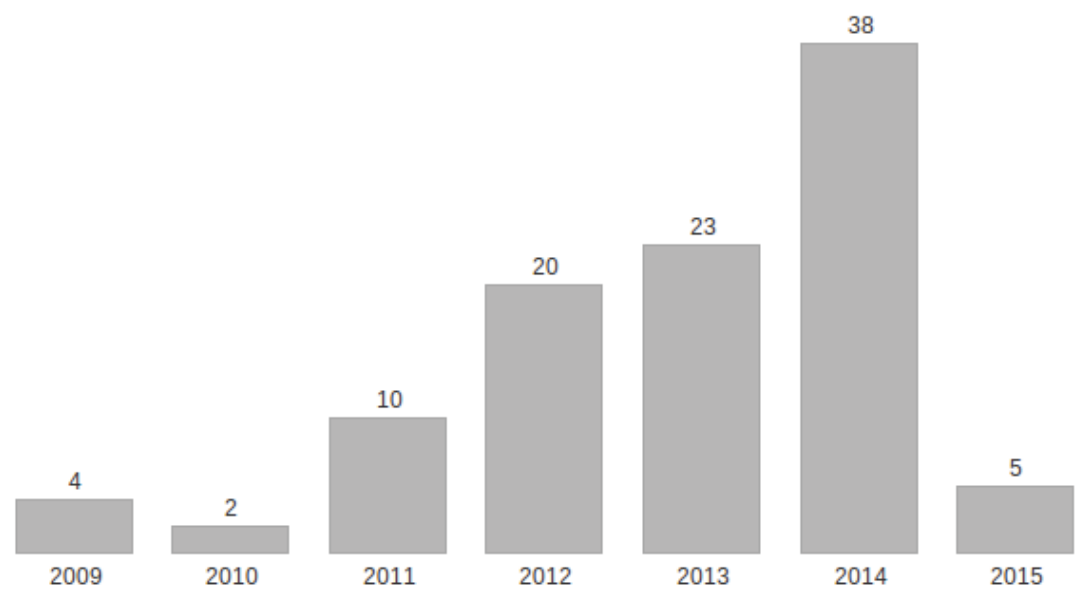

Fuente: Elaboración propia/Marzo de $2015^{2}$

Las tecnologías cívicas en España se caracterizan por su falta de exclusividad temática. Esto implica que, bajo el mandato de una mejora democrática y de la necesidad de información sobre el funcionamiento del sistema se entremezclen soportes y temas. De este modo encontramos la misma aplicación en diversos bloques: ámbitos con una voluntad clara de transparencia (Tu derecho a saber ${ }^{3}$ ), comprensión del funcionamiento de las instituciones (Qué hacen los diputados, El teu Parlament) rendición de cuentas (Qué hacen los diputados, Quién Manda, Transparencia de las cuentas públicas, Graba tu pleno, Sueldos públicos), generación de información para periodistas y ciudadanía (España en Llamas, El Indultómetro, Analiza.info) implicación en medidas legislativas (Kuorum) participación en procesos políticos (Ágora Voting) o descentralización de redes sociales (VirtualPol, N-1) (Magallón, 2014).

La profusión de herramientas cívicas destinadas a la mejora democrática pueden interpretarse desde dos puntos de vista. El primero enlaza con la necesidad de fiscalización por parte de la ciudadanía de la actividad de los gobiernos ante la falta de cultura de rendición de cuentas. La segunda destaca la falta de iniciativas diseñadas por las administraciones, así como su escasa implicación en este ámbito. Más allá de algunos proyectos en instituciones pequeñas que cuentan con aplicaciones móviles para que la ciudadanía reporte incidencias sobre los servicios públicos habría que destacar excepciones como las del portal de datos del Ayuntamiento de Zaragoza (en el que se muestran los presupuestos municipales, las empresas licitadoras, etc.) o los portales de los Gobiernos de Aragón y País Vasco ${ }^{4}$.

Derivado de esta falta de implicación por parte de las administraciones públicas las tecnologías cívicas adolecen de cierta precariedad. La dependencia económica de mecenazgos e iniciativas de crowdfunding han condicionado cierta invisibilidad de los proyectos limitándolos a un uso reducido. Su planificación y desarrollo parte 
de organizaciones en las que la militancia (previa y presente) vehiculan la razón de ser de las tecnologías propuestas restando cierto crecimiento y profesionalismo a los proyectos. Esto no resta valor a la voluntad de los impulsores por emprender propuestas en un ámbito sin mucho desarrollo en España, aunque existe el riesgo de limitarse al consumo de un usuario más intensivo y militante.

El reto de futuro de las tecnologías cívicas pasaría por establecer tres tipos de alianzas con los tres actores que se desenvuelven en la esfera pública. La primera de ellas se centraría en demandar la implicación de las administraciones en el desarrollo de proyectos de este tipo, más allá de repositorios con la información municipal y la rendición de cuentas obligatoria. El objetivo final pasaría por ampliar la democracia con un uso político de la tecnología estableciendo vínculos estables y de calidad con la ciudadanía. Esta segunda alianza se orientaría a potenciar su implicación en estos proyectos situándolos no como espectadores del proceso democrático sino como colaboradores activos y cocreadores del mismo. Por último, y a camino de las otras dos alianzas, el papel de los medios de comunicación sería vital no solo como herramienta pedagógica y de mediación efectiva entre gobernantes y gobernados, sino también como elemento redefinidor del papel del periodista en el contexto digital.

\section{Periodismo y tecnologías cívicas}

La presencia de la sociedad civil en los medios de comunicación se ha caracterizado tradicionalmente por su escasa visibilidad. En un estudio sobre el contenido de los cibermedios generalistas en España solo en un 18\% de las ocasiones estas organizaciones eran consideradas como fuentes informativas, frente al $45,1 \%$ de actores gubernamentales y administraciones públicas (Odriozola, 2012).

Su presencia se limita habitualmente a momentos puntuales como la presentación de un informe, la elaboración de una denuncia, la celebración de una campaña de movilización o como fuente complementaria a una noticia o reportaje aportando conocimiento experto. Ante esta tendencia muchas organizaciones reclaman espacios mediáticos con la realización de acciones llamativas o transgresoras que intentan romper la linealidad de acceso informativo habitual pero que suponen una presencia anecdótica.

Sin embargo, es cada vez más habitual cierta incursión de los marcos discursivos de la sociedad civil en los medios debido a un cambio en los repertorios de acción colectiva de las organizaciones y al surgimiento de nuevos medios de comunicación digital. A estos dos rasgos se une el desarrollo de tecnologías cívicas que condicionan cierta redefinición de las relaciones entre periodistas y sociedad civil y sitúan a las organizaciones en un plano de visibilidad como fuentes informativas. Podemos distinguir tres tipos de categorías en esta relación: 
- Fuentes que proveen información sensible: Desarrollo de aplicaciones seguras que reciben informaciones y datos sensibles de fuentes que no se pueden revelar. En esta categoría podrían ubicarse Fíltrala ${ }^{5}$ (plataforma ciudadana de denuncia independiente surgida en 2014) y Buzón $X^{6}$ (ligada a Xnet y también existente desde el mismo año).

Ambos proyectos colaboran activamente con medios. En el caso de Filtrala tiene establecida una colaboración con Diagonal, La Marea, Eldiario.es, Mongolia, Crític y Directa. Por su parte Buzón X colabora con Antena 3, EFE, Cadena Ser o El Confidencial. El precedente de estas dos iniciativas fue el proyecto Minileaks surgido en 2011 y disuelto en 2013 por la dificultad de hacer sostenible una plataforma centrada en recibir información sensible de denuncia ${ }^{7}$.

- Colaboraciones por especialización temática: Miembros de organizaciones que a partir del desarrollo de tecnologías cívicas participan en los medios de comunicación no obteniendo cobertura sino accediendo de manera directa.

En esta categoría se distinguen casos como eldiario.es que ofrece de manera periódica su blog Zona Crítica como tribuna abierta para organizaciones y activistas (como por ejemplo Access Info Europe). De igual modo, la Fundación Ciudadana Civio (Quién manda, Tu derecho a saber, El Indultómetro) colabora habitualmente en la Cadena Ser; Sueldos Públicos y Maldita Hemeroteca cuentan con secciones fijas en el programa de televisión El Objetivo de La Sexta y este último, a su vez y de manera asidua, en eldiario.es.

- Herramientas como fuentes informativas para los medios: aplicaciones desarrolladas por organizaciones de la sociedad civil con datos públicos y que son empleadas como fuentes para desarrollar noticias y reportajes. En este plano destacan iniciativas como España en llamas, Dónde van mis impuestos y El BOE nuestro de cada día (Civio) o Qué hacen los diputados (Asociación Qué hacen).

Es preciso subrayar que en muchas de estas iniciativas la propiedad intelectual no es exclusiva de los promotores del proyecto, sino que emplean licencias libres. Esta forma de protección de los contenidos, por la que se reservan únicamente algunos derechos, permite la replicación de artículos en medios de comunicación. Algunos nuevos medios de información generalista digitales han optado por incorporar licencias libres, lo que les permite compartir en igualdad de condiciones los contenidos generados por los proyectos de la sociedad civil a partir de sus propias tecnologías. Algunos ejemplos son eldiario.es, La Marea o Zoom News. 


\section{Conclusiones}

El desarrollo de las tecnologías cívicas y el surgimiento de medios de comunicación digitales inauguran un nuevo ámbito de relación entre los actores de la esfera pública. La comunicación entre gobernantes y gobernados puede resultar más eficaz suprimiendo intermediarios tradicionales y mejorando la rendición de cuentas y la fiscalización del poder. Su consecuencia más inmediata sería una mejora democrática en términos de control e inclusión de la ciudadanía en los procesos de gestión y decisión públicos.

La potencialidad de las tecnologías cívicas reside en aumentar la información facilitando herramientas de participación y empoderamiento de la ciudadanía. Sin embargo, es preciso trabajar en una cultura de uso y desarrollo de estos proyectos no solo de cara a los gobernantes. Resulta vital implementar métodos de inclusión de una mayor parte de la ciudadanía en el proceso de cocreación de estas herramientas. No solo como usuarios o financiadores, sino como potenciales informantes-desarrolladores de las iniciativas.

Por otra parte es preciso fortalecer las alianzas con los medios de comunicación reelaborando un patrón constante de relación a través del uso de los datos derivados de las tecnologías cívicas, estableciendo colaboraciones por especialización temática y proporcionando información sensible y susceptible de ser difundida. Estos protocolos pueden provocar una excesiva connivencia entre las organizaciones y los medios de comunicación convirtiéndose en cajas de resonancia polarizadas y fragmentando el discurso público. Sin embargo, podrían suplir las continuas exclusiones de la sociedad civil en la esfera pública proporcionándoles acceso y cobertura de manera directa.

Por último, y como contexto de desarrollo de las tecnologías cívicas, sería necesario atender a las estructuras de oportunidad política (en términos de oportunidad/ restricción para quien plantea la acción y facilitación/represión por parte de las autoridades) (Tilly, 2009). La desconfianza en las instituciones políticas y sus gestores (Lobera, 2015: 99), así como el contexto de repolitización en forma de nuevos modos de participar tras el 15-M, apuntan a ciertas oportunidades para el desarrollo y la implementación de tecnologías cívicas. Sin obviar los riesgos de experimentar un proceso de cooptación se aprecia un contexto más abierto, participativo y proclive a la innovación ciudadana y periodística.

\section{Referencias bibliográficas}

ANDERICA, V. (2014). "Retos comunicativos de la sociedad civil: Del lobby social a la agenda pública". En: ESPÍRITUSANTO, Ó. (dir.). Tecnologías cívicas y participación ciudadana. Revista de Estudios de Juventud, nº 105. p. 71-90. 
ANDERSON, C.W.; BELL, E.; SHIRKY, C. (2012). Post-Industrial Journalism: Adapting to the Present. USA. The Tow Center.

BENKLER, Y. (2014). La riqueza de las redes. Cómo la producción social transforma los mercados y la libertad. Barcelona: Icaria.

BOLAÑOS, V.; ANDERICA, V.; MAGALLÓN, R.; SÁNCHEZ DUARTE, J. M. (2015). Evolución y desarrollo de las tecnologías cívicas en España. Entre el activismo y el periodismo. XXI Congreso Internacional de la SEP. 19 y 20 de junio de 2015.

BRUNS, A. (2003). "Gatewatching, not gatekeeping: Collaborative online news". En: Media International Australia Incorporating Culture and Policy: quarterly journal of media research and resources, $\mathrm{n}^{\circ}$ 107. p. 31-44.

CHRISTENSEN, H. S.; BENGTSSON, A. (2011). "The political competence of internet participants". En: Information, Communication \& Society, $\mathrm{n}^{\mathrm{o}}$ 14:6. p. 896-916.

DAHLBERG, L. (2007) "Rethinking the fragmentation of the cyberpublic: from consensus to contestation". En: New Media Society, n 9. p. 827.

DAHLGREN, P. (2005). "The Internet, Public Spheres, and Political Communication: Dispersion and Deliberation" En Political Communication. 22: p. 147-162.

DÍAZ NOSTY, B. (1995). "La preagenda de los medios, expresión de la matriz mercantil de la comunicación”. En: Tendencias. Madrid: Fundesco.

FLANAGIN, A.; STOHL, C.; BIMBER, B. (2006). "Modeling the Structure of Collective Action". En: Communication Monographs, $\mathrm{n}^{\circ}$ 73:1. p. 29-54.

GOLDBERG, G. (2010). "Rethinking the public/virtual sphere: The problem with participation". En: New Media Society, n 13. p. 739.

GROUNLUND, I.; STRANDBERG, K.; HIMMELROOS, S. (2009). "The challenge of deliberative democracy online - A comparison of face-to-face and virtual experiments in citizen deliberation". En: Information Polity, n ${ }^{\circ}$ 14. p. 187-201.

HABERMAS, J. (2004). Historia y crítica de la opinión pública. Buenos Aires: Gustavo Gilli.

IOSIFIDIS, P. (2011). "The public sphere, social networks and public service media". Information, Communication \& Society, $\mathrm{n}^{\circ}$ 14:5, p. 619-637.

LANIER, J. (2011). Contra el rebaño digital. Un manifiesto. Barcelona: Debate.

LOBERA, J. (2015). "De movimientos a partidos. La cristalización electoral de la protesta". Revista Española de Sociología, no 24, p. 97-105.

LOADER, B. D.; MERCEA, D. (2011): “Networking Democracy?”. En: Information, Communication \& Society, $\mathrm{n}^{\circ}$ 14:6. p. 757-769.

MACASSI, S. (2010). "Medios y conflictos sociales entre el rating y el activismo". En: Diálogos de la comunicación, $\mathrm{n}^{\circ} .81$.

MAGALLÓN, R. (2014). "Tecnologías cívicas y participación ciudadana”. En ESPÍRITUSANTO, Ó. (dir.) (2014). Periodismo Ciudadano. Nuevas formas de comunicación, organización e información. Revista de Estudios de Juventud, nº 105. p. 53-70.

MANCINI, P. (2013). "Media Fragmentation, Party System, and Democracy". En: The International Journal of Press/Politics, $\mathrm{n}^{\circ} 18$ (1). p. 44-60. 
MASIP, P.; SUAU, J. (2014). "Audiencias activas y modelos de participación en los medios de comunicación españoles". En: Hipertext.net [online], $\mathrm{n}^{\mathrm{0}} 12$.

MOZOROV, E. (2011). The netdesilusion. The dark side of Internet freedom. Publica Affairs.

ODRIOZOLA, J. (2012). "Análisis de contenido de los cibermedios generalistas españoles. Características y adscripción temática de las noticias principales de portada". En: Comunicación y sociedad, 25, no 2. p. 279-304.

PATEL, M.; STOCKY, J.; GOURLEY, S.; HOUGHTON, D. (2013). The Emergence of Civic Tech: Investments in a Growing Field. Knight Foundation.

POLITIKON (2014). La urna rota. La crisis política e institucional del modelo español. Barcelona: Debate.

ROBERTSON, S. P.; VATRAPU, R. K.; MEDINA, R. (2010). "Off the wall political discourse: Facebook use in the 2008 U.S. presidential election". En: Information Polity, n ${ }^{\circ}$ 15. p. 11-31.

ROTMAN, D.; PREECE, J.; VIEWEG, S. (et. Al) (2011). "From Slacktivism to Activism: Participatory Culture in the Age of Social Media". CHI 2011, May 7-12, 2011, Vancouver, BC, Canada. Proceedings of the 2011 annual conference extended abstracts on Human factors in computing systems. p. 819-822.

SAMPEDRO, V. (2000). Opinión Pública y democracia deliberativa. Medios, sondeos y urnas. Madrid: Istmos.

SAMPEDRO, V.; SÁNCHEZ-DUARTE, J.M.; CAMPOS, E. (2014). "Participación ciudadana en las campañas electorales. Debates teóricos y una aproximación tipológica". En: COTARELO, R.; OLMEDA, J.-A. (coords.) (2014). La democracia del siglo XXI. Política, medios de comunicación, internet y redes sociales. Madrid: Centro de Estudios Políticos y Constitucionales.

SOUSA, H.; PINTO, M.; COSTA E SILVA, E. (2013). "Digital public sphere: weaknesses and challenges". En: Comunicação e Sociedade, no 23. p. 9 - 12.

SUNSTEIN, R. C. (2007). Republic.com. 2.0. Princeton, NJ: Princeton University Press.

TABARES, E. (2000). "Medios de comunicación, ¿instrumentos para la solidaridad?". En: Comunicar: Revista científica iberoamericana de comunicación y educación, $\mathrm{n}^{\circ}$ 15. p. 37-44.

TARROW, S. (2011). "El poder en movimiento. Los movimientos sociales, la acción colectiva y la política". Madrid: Alianza Editorial.

TILLY, C. (2009). Los movimientos sociales. 1768-2009. Madrid: Crítica.

TREJO, A. (2013). Comunidades de Tecnología Cívica o la Inteligencia de la Cooperación TIC entre ciudades. Observatorio Cenatic.

VAN LAER, J.; VAN AELST, P. (2010). "Internet and social movement action repertoires. En: Information, Communication \& Society, n ${ }^{\circ}$ 13:8. p. 1146-1171.

ZÚÑIGA, H. (2011). Social Media Use for News and Individuals' Social Capital, Civic Engagement and Political Participation. Centro de Investigación en Comunidad, Periodismo y Comunicación (CJCR) de la Escuela de Periodismo de la Universidad de Austin, Texas. 


\section{Notas}

1. El proyecto Discursia (www.discursia.com) creado en 2007 intentó acercar la información institucional del Parlamento a los usuarios. Era un directorio de diputados del Congreso que tuvo un impacto discreto y en el que a través de una web los usuarios podían conocer la actividad de los parlamentarios, datos de salarios, contacto, etc.

2. La elaboración de este gráfico se ha realizado a partir de una recogida propia de datos en los últimos años ante la inexistencia de un repositorio oficial o investigaciones previas en España sobre este tema de estudio. Para su configuración se han tenido en cuenta las herramientas, páginas y aplicaciones digitales que siguen online y que han sido creadas por organizaciones de la sociedad civil, instituciones a nivel local, regional y nacional, empresas y organizaciones privadas que cumplen con alguno de los objetivos mencionados en el anterior epígrafe. Asimismo, se han considerado como tecnologías individuales aquellas aplicaciones móviles basadas en diferentes sistemas operativos (iOS y Android).

3. Esta iniciativa forma parte de los múltiples proyectos impulsados y desarrollados por la Fundación Civio (El Indultómetro, España en llamas, Quién Manda...) una de las organizaciones más importantes en el ámbito de las tecnologías cívicas en España (www.civio.es) [consultada el 30/06/2015]

4. Cabe destacar en este punto las carencias del portal de transparencia impulsado por el Gobierno de España en 2014. A diferencia de los portales de estas dos Comunidades Autónomas el portal del Gobierno no permite la reutilización de los datos.

5. www.filtrala.org [consultada el 30/06/2015]

6. www.xnet-x.net/buzonx [consultada el 30/06/2015]

7. www.mini-leaks.com/leaks/cierre-de-minileaks/ [consultada el 30/06/2015] 